\title{
Control of a variable-geometry suspension as independent wheel steering
}

\author{
Balázs Németh, Dániel Fényes and Péter Gáspár \\ Institute for Computer Science and Control, Hungarian Academy of Sciences \\ E-mail: balazs.nemeth@sztaki.mta.hu
}

\begin{abstract}
The paper presents the modeling and control design of a variable-geometry suspension system, which is applied as independent wheel steering for in-wheel electric vehicles. The variablegeometry suspension modifies the tilting of the wheel, which has an effect on the steering of the wheels through the variation of the scrub radius. In the paper the model of the suspension-steering system and the relationship between the steering and the suspension geometry are formulated using nonlinear dynamical equations. The model is validated through a high-fidelity multi-body suspension model SimMechanics. In the paper the robust combined feedforward-feedback control design of the independent steering system is presented. Finally, the proposed control system is used in different vehicle scenarios.
\end{abstract}

Topic / Vehicle dynamics, Suspension, Active safety and driver assistance systems

\section{INTRODUCTION}

The in-wheel electric drive offers new challenges in the steering of the vehicle, such as independent steering. The goal of the independent steering concept is to improve the lateral dynamics of the vehicle using individually controlled wheels. The independent steering control for the rear wheels to modify the toe angle is presented by [1]. An indirect power steering measure called differential drive torque assisted steering was proposed by [2]. A fault-tolerant control approach for a four-wheel independently actuated electric vehicle was proposed by [3].

In this paper an independent steering is proposed, which is based on the variable-geometry suspension solution, see [4]. The aim of the suspension control is the modification of the geometry, which results in a change in the camber or the toe angle. A rearsuspension active toe control for the enhancement of driving stability is proposed by [5]. In the paper the control design of an independent wheel steering system for the front wheels is proposed. The novelty of the paper is the application of the variable-geometry suspension in the steering solution. The contributions are the modeling and validation of the suspension system, the control design of the actuator and the robust trajectory tracking control of the vehicle. In the control solution the actuator and the tracking control are connected in a hierarchical structure.

\section{MODELING OF THE COMPONENTS}

The goal of the variable-geometry suspension is to perform the wheel camber angle and the scrub radius modification. The camber angle results in a lateral force on the tire-ground contact. Since the longitudinal force has a rotator effect on the wheel, the scrub radius of the wheel influences the steering dynamics of the wheel. Therefore, a lateral force from the wheel steering through the scrub radius modification is resulted.

Since the variable-geometry suspension has one actuator in each wheel, it is necessary to find a suspension construction by which the lateral force generation of the camber and the scrub radius is in coordination. Thus, the forces from the wheel tilting and the steering from the scrub radius have the same effect on the vehicle dynamics. The scheme of the variablegeometry suspension is shown in Figure 1.

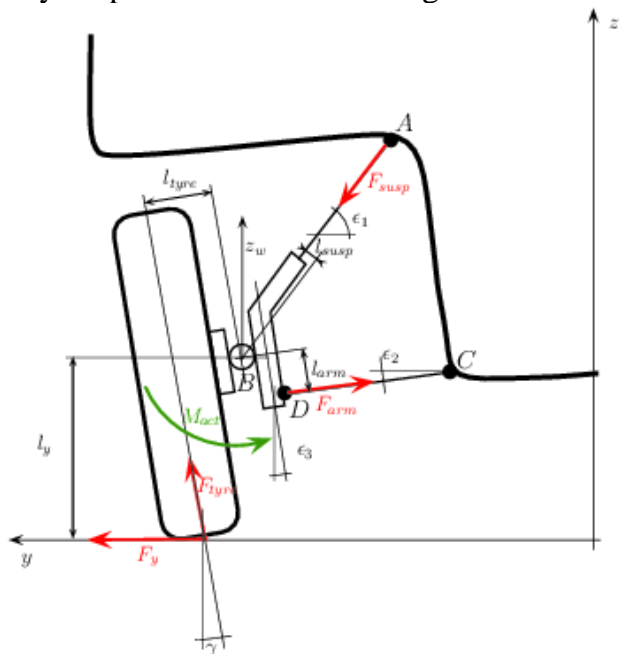

Fig. 1 Scheme of the suspension construction

The actuator is incorporated in the suspension between the wheel hub and the wheel. It is able to generate an active torque $M_{\text {act }}$ around $B$ to tilt the wheel. In the McPherson construction the suspension is able to rotate around the connection point $A$ of the chassis. Moreover, the arm connects the hub $D$ and the chassis $C$ with joints, which are able to guarantee the rotation and the motion of the suspension.

Several forces influence the motion of the suspension and the wheel, such as the suspension and tire compression/damping forces, the lateral tire-road force, forces on the suspension arms. The motion of the suspension is described by three dynamic equations: the vertical motion of the wheel hub, the torque on the suspension is described to derive the rotation of the wheel hub, and the tilting of the wheel.

The lateral motion of the vehicle is influenced by the modification of the camber angle. Moreover, the camber angle influences the scrub radius of the wheel, 
which results in wheel steering. The lateral dynamics of the vehicle is influenced in two ways: the wheel camber angle and the steering angle have lateral force effects. The lateral motion of the vehicle is described by the bicycle model.

\section{ROBUST CONTROL DESIGN}

The goal of the independent steering is to control the lateral motion of the vehicle through the wheel camber angle modification. It requires the solutions of two control problems. First, the lateral performance of the vehicle by appropriate wheel camber angles must be guaranteed. Second, the camber angles of the wheels through the variable-geometry suspension actuation must be realized. Thus, in the following the solutions of these control problems in a hierarchical structure are proposed.

The goal of the lateral control design is to guarantee the trajectory tracking of the vehicle. It means that the vehicle must follow a reference yaw-rate signal $\dot{\psi}_{\text {ref }}$, which is computed using the velocity and the steering wheel angle of the driver. Since the suspension actuator has uncertainties and the sensor measurement is noisy, a the robust $\mathrm{H}_{\infty}$ strategy is applied for the tracking control design. The objective of this method is to minimize the $\mathrm{H}_{\infty}$ norm of the gain from the disturbances to the performances. The objective of the variable-geometry suspension control is to realize the camber angle, which is yielded by the robust controller. Therefore, a Linear Quadratic (LQ) control for the wheel tilting actuation is designed. In the LQ control design the aim is to minimize a quadratic cost function, which incorporates the camber angle tracking performance, the active torque $M_{\text {act }}$ and design weights.

In Figure 2 the architecture of the lateral control and the variable-geometry suspension control are presented. It illustrates the cooperation of the different controllers. The lateral dynamic control computes the required left and right wheel camber angles using the yaw-rate error. The variable-geometry suspension control generates the actuation torques, by which the steering of the wheels is realized. Thus, the lateral motion of the vehicle is improved.

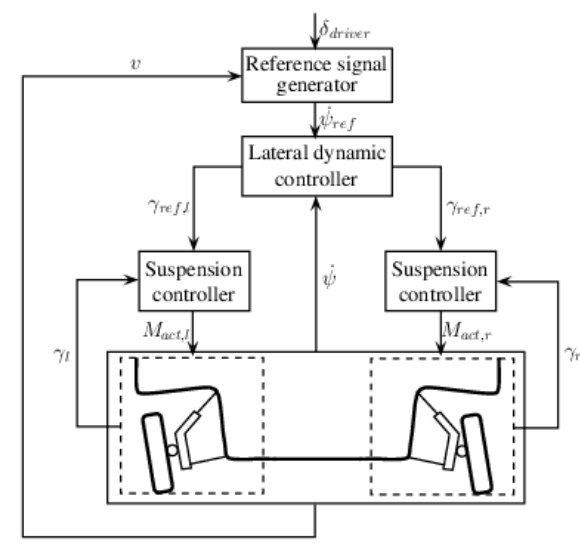

Fig. 2 Architecture of the control systems

\section{SIMULATION RESULTS}

In the followings a $\dot{\psi}_{\text {ref }}$ tracking problem is presented briefly. The yaw-rate tracking of the vehicle is shown in Figure 4(a). It can be seen that the vehicle is able to guarantee the precise tracking of the $\dot{\psi}_{\text {ref }}$ signal. It results from the appropriate coordination of the lateral dynamic controller and the variable-geometry suspension control. Figure 4(b) illustrates the required and the real steering angle of the front right wheel. The simulation shows that the variable-geometry suspension is able to realize the required steering angle, which is achieved by the camber angle modification at the right, see Figure 4(c). The camber angle illustrates that the low-level suspension control is sufficiently accurate to guarantee the required actuation which is commanded by the high-level controller, see Figure 4(d).

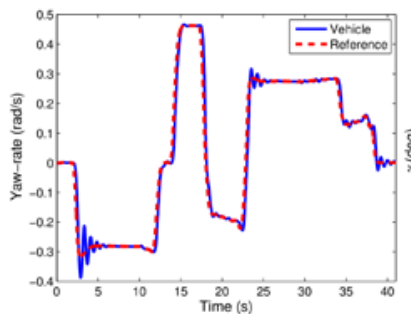

(a) Yaw-rate tracking

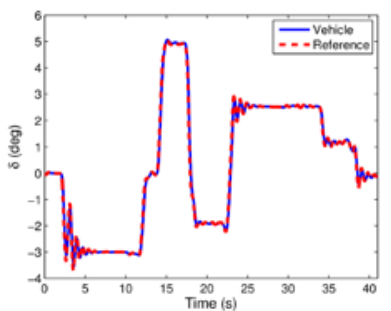

(c) Wheel camber

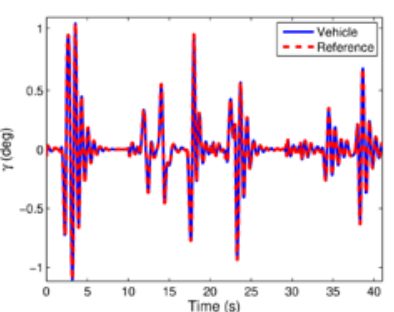

(b) Wheel steering

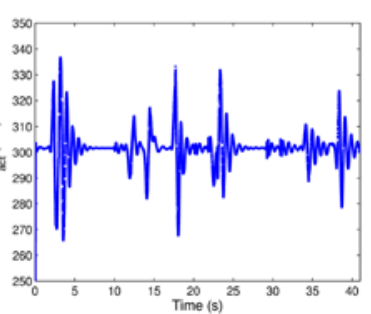

(d) Actuation
Fig. 3 Simulation results

\section{CONCLUSIONS}

The paper is proposed an alternative way to independent steering control. Since in-wheel electric vehicles are likely to have a significant impact in the future, further research on the variable-geometry suspension control is reasonable. In the paper the modeling and control of independent steering for an inwheel electric vehicle have been proposed. The nonlinear formulation of the suspension system, together with the modeling of the lateral dynamics is presented. The main contribution of the paper is a hierarchical control architecture, which incorporates the robust $\mathrm{H}_{\infty}$ control of the trajectory tracking and the LQ optimal design of the wheel tilt positioning.

\section{REFERENCES}

[1] H.S. Lee, U.K. Lee, S.K. Ha, and C.S. Han. Fourwheel independent steering system for vehicle handling improvement by active rear toe control. JSME Int. J. Ser. C, 42(4):947-956, 1999.

[2] J. Wang, Q. Wang, L. Jin, and C. Song. Independent wheel torque control of electric vehicle for differential drive assisted steering. Mechatronics, 21:63-76, 2011.

[3] C. Hu, H. Jing, R. Wang, F. Yan, and N. Chen. Fault-tolerant control of fwia electric ground vehicles with differential drive assisted steering. 9th IFAC Safeprocess Symposium, Paris, 2015.

[4] W.J. Evers, A. van der Knaap, I. Besselink, and H. Nijmeijer. Analysis of a variable geometry active suspension. Int. Symp. Advanced Vehicle Control, Kobe, 2008.

[5] A. Goodarzia, E. Oloomia, and E.Esmailzadehb. Design and analysis of an intelligent controller for active geometry suspension systems. Vehicle System Dynamics, 49(1):333-359, 2010. 